\title{
Angular velocity discrimination
}

\author{
MARY K. KAISER \\ NASA Ames Research Center, Moffett Field, California
}

\begin{abstract}
Three experiments were designed to investigate naive observers' abilities at discriminating the rotational velocities of two simultaneously viewed objects. In Experiment 1, rotations could occur about parallel or orthogonal axes, with initial orientations in phase or out of phase, and (for parallel rotational axes) in the same or opposite direction. Differential thresholds were approximately $10 \%$. In Experiment 2, stimulus objects differed in the number of faces revealed in rotation (three vs. four). Observers' response curves had no greater spread, but their PSEs (points of subjective equality) were shifted such that there was a partial compensation for faces revealed per unit time. In both Experiment 1 and Experiment 2, performance was consistent across rotational axis and directional conditions. In Experiment 3, the effect of object size was examined, in order to determine the extent to which angular velocity judgments are influenced by the tangential velocity of the faces. When the comparison cube's edges were half the length of the standard's, PSEs were elevated $18.5 \%$. Taken together, these data suggest that observers are able to discriminate angular velocities with a competence near that for linear velocities. However, perceived angular rate is influenced by structural aspects of the stimuli.
\end{abstract}

Object motion can be parsed into two general categories: translations and rotations. One critical issue in the perception of object motion is the perception of velocity. A perceptual competence for rotational velocity is presupposed by several structure-from-motion algorithms, particularly those based on dynamic contour deformation (Cortese \& Andersen, 1989; Pollick, 1989). In addition, my colleagues and I have argued that observers are unable to perform dynamical analyses on angular kinematics because these motions have a different perceptual function, that of form specification (Proffitt, Kaiser, \& Wheelan, in press). However, it may be the case that people are insensitive to angular dynamics simply because they lack the ability to perceive angular kinematics with any reasonable level of quantitative precision. Whereas there is a robust psychophysical literature on translational velocity perception (Lappin, Bell, Harm, \& Kottas, 1975; McKee, 1981), little attention has been given to the perception of angular velocities. This is unfortunate since, in addition to the theoretical concerns listed above, rotational motions have several unique properties that can provide new insights into velocity perception.

The first property of interest is the decoupling of time and distance. In rotation, an object's position in space is not a linear function of time. In fact, the object's initial orientation is regained after each $360^{\circ}$ of rotation. Thus,

Portions of this paper were presented at the annual spring meeting of the Association for Research in Vision and Ophthalmology, Sarasota, Florida, May 1989, and at the Fifth International Conference on Event Perception and Action, Oxford, Ohio, July 1989. I thank Larry Beck of Sterling Software for his programming support, and Walter Johnson, John Perrone, Mike Braunstein, and two anonymous reviewers for their helpful comments on earlier versions of this manuscript. Requests for reprints should be sent to Mary K. Kaiser, NASA Ames Research Center, Mail Stop 239-3, Moffett Field, CA 94035. unlike linear motions, displacement is not a direct correlate of motion duration. This eliminates the possibility of observers' utilizing relative displacement information as the basis of their velocity discriminations.

Second, an object's angular velocity is not specified by the tangential velocity of any single element (or even weighted average of elements) on the object's surface. Given a large and small object rotating with equal angular velocities, elements from the large object will have greater 3-D object (and projected 2-D image) velocities than those from the small object. Angular velocity is thus a higher order construct, defined by relational quantities rather than absolute velocities.

Finally, there is the potential for object properties, such as shape, to influence perceived rotational rate. For example, an object with more facets will reveal more faces as it undergoes rotation. These face transitions provide a disruptive temporal frequency cue, which could bias perceived velocity.

This paper describes a set of three experiments on rotational velocity discrimination. Rotations in these experiments were constrained to occur about the $x$ - and $y$-axes, resulting in linear 2-D image trajectories. Presumably, such motions are processed by the same early vision mechanisms that analyze translational velocities. (The perception of $z$-axis rotations would involve rotary motion detectors, whose response characteristics are not as well understood or defined [Sakata, Shibutani, Ito, \& Tsurugai, 1986].) This similarity of image motion components makes relevant the comparison of rotational and translational velocity discrimination competencies.

\section{EXPERIMENT 1}

Experiment 1 was designed to examine observers' abilities to discriminate the angular velocity of two identical 
forms under a variety of rotational conditions. If angular velocity discrimination depends on a low-level matching strategy, performance should decline when rotations occur in opposite directions, about parallel axes, or with the objects out of phase.

\section{Method}

Observers. Eight observers ( 4 males and 4 females) participated in all three experiments. Their ages ranged from 19 to 25 years. All had normal or corrected-to-normal binocular vision. Prior to participation, each observer was informed of the general nature of the research. The order in which the experiments were completed was randomized across observers to avoid systematic practice effects.

Apparatus and Stimuli. The experimental program was run on a Silicon Graphics IRIS 3130 workstation. Stimuli were displayed on a 1,280 × 1,024 pixel, $48.25-\mathrm{cm}$ (diagonal) RGB monitor with a $60-\mathrm{Hz}$, noninterlaced raster refresh rate. Displays were updated at 30 frames/sec. Angular velocity was varied by altering the degree of angular displacement between frames. The viewing geometry used in the experiments is shown in Figure 1. The station point for the display was elevated slightly $\left(10^{\circ}\right)$ above the $z$-axis in order that more of the objects' 3-D structure be visible. (Preliminary observations indicated that pure $z$-axis viewing resulted in the stimuli's appearing as transforming 2-D forms.) At the prescribed viewing distance, the entire display window subtended approximately $15^{\circ}$ of visual angle. The two stimulus forms were displayed side by side. Each object subtended no more than $5^{\circ}$. The observers' task was to select which of the two forms appeared to rotate faster. The experiment was conducted with minimal ambient lighting; the frame of the monitor and surrounding furniture were clearly visible.

The stimulus forms and the rotational motion conditions for Experiment 1 are depicted in Figure 2a. The stimuli were simulated solid cubes with the faces pseudoshaded - that is, each face was assigned a luminance value, which it retained throughout the rotation. (In a pilot study, the faces of the cube were color-coded. However, observers reported that they employed color-matching strategies. Hence, these color cues were removed in Experiment 1. No observer reported using a face-matching strategy with the pseudoshaded stimuli.) The cubes' proximal edges projected $4.5^{\circ}$ in length. The standard cube rotated at $.33 \mathrm{~Hz}$. The comparison cube rotated about an axis that was either parallel or orthogonal to the standard's. If the axis was parallel, rotation was in either the same or the opposite direction. This yielded three axis conditions: parallel same, parallel opposite, and orthogonal. Rotations occurred with the standard and comparison forms either initially in phase or $45^{\circ}$ out of phase.

Design. Observers were asked to make relative rotational velocity judgments for each stimulus pair. Transformed up-down stair- cases with decreasing step sizes (Levitt, 1971) were used to track observers' $X_{29.3}$ and $X_{70.7}$ (i.e., the velocities at which the comparison stimulus was judged faster $29.3 \%$ and $70.7 \%$ of the time) for each motion condition. The twelve staircases ( 3 axes conditions $\times 2$ phase conditions $\times 2$ tracking points) were interlaced to ensure independence of responses for adjacent trials of each staircase.

Procedure. Observers were seated at the correct viewing position for the projection geometry, although head and eye position were unconstrained. On each trial, an auditory warning sounded, and the standard and comparison stimuli were displayed simultaneously for $4 \mathrm{sec}$. At the termination of the display sequence, the response menu was highlighted; observers indicated which form appeared to rotate faster. The position of the standard (left vs. right) varied from trial to trial. Observers were not given feedback during the experiment. About $\mathbf{4 5} \mathrm{min}$ were required for an observer to complete Experiment 1 .

\section{Results}

For each condition, $X_{29.3}$ and $X_{70.7}$ were determined for observers by taking the mean of their midrun estimates. The difference of these two points is equal to $1.09 \sigma$, so a multiplier of 0.619 was used to obtain an estimate of the differential threshold (DT). The average of these two points was used to estimate observers' point of subjective equality (PSE). (This latter estimation procedure requires a symmetry assumption for the underlying psychometric function.)

Figure 3 shows the DT data averaged across observers. (Bars indicate 1 SEM.) An analysis of variance (ANOVA) indicates that DTs were not affected by rotational axes (parallel vs. orthogonal), rotational direction (same vs. opposite), or phase relation (in-phase vs. out-of-phase). Pilot data had indicated an effect for phase, but this appears to have been an artifact of the coloring of the stimulus cubes; observers were able to employ color-matching strategies for in-phase conditions. The PSE data are presented in Figure 4 (bars indicate $1 S E M$ ). For all conditions, PSEs did not differ significantly from the point of objective equality (POE).

\section{EXPERIMENT 2}

In Experiment 2, the effect of object structure on perceived angular velocity was examined. In particular, the number of faces revealed in rotation was manipulated. It

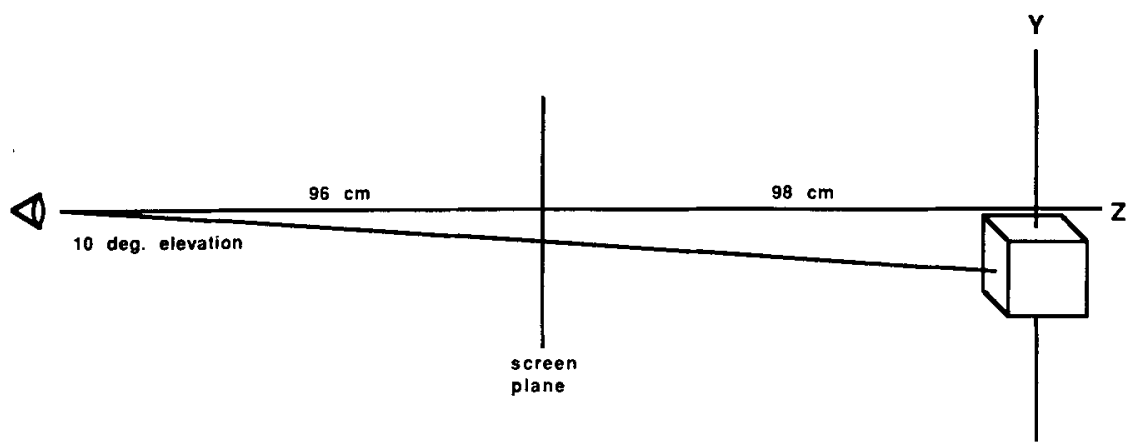

Figure 1. Viewing geometry employed in Experiments 1, 2, and 3. System is shown along the $x$-axis, with the standard stimulus cube depicted (not to scale). 
(a)

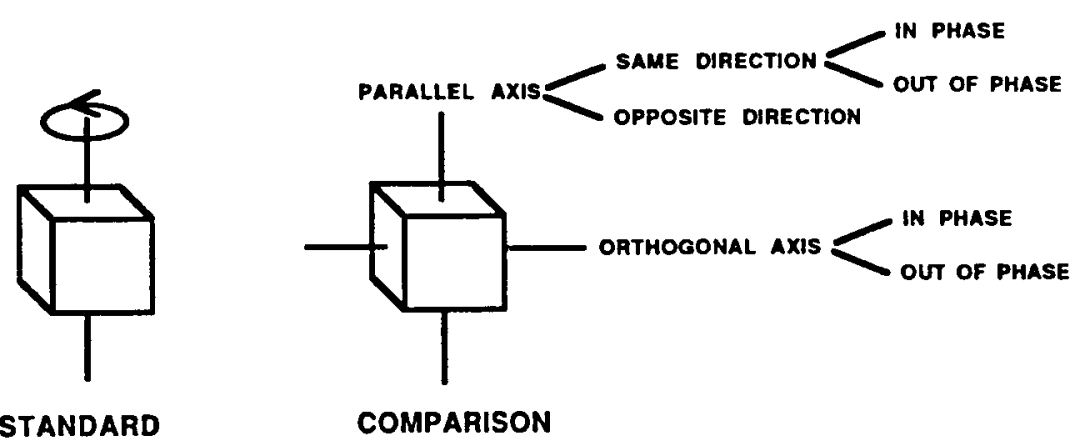

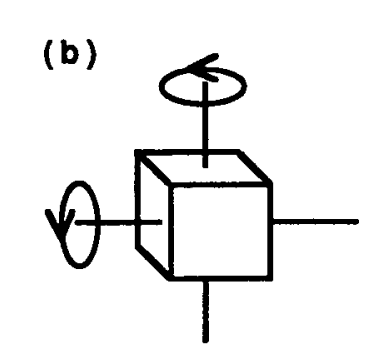

STANDARD

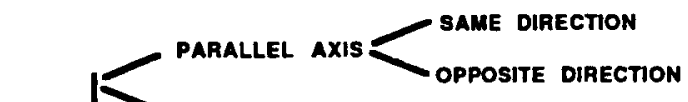

MOMPARISON

(c)

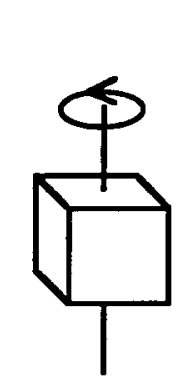

STANDARD

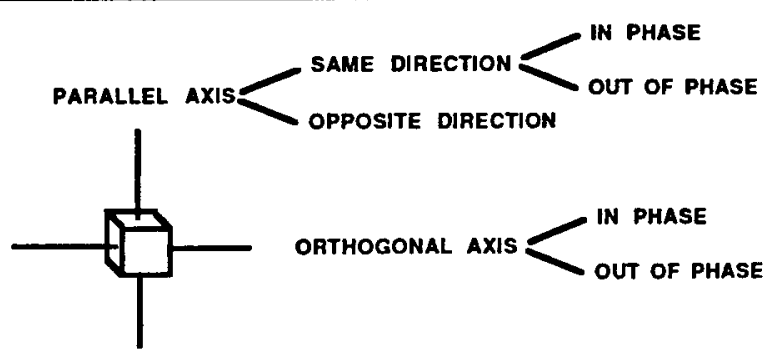

COMPARISON

Figure 2. Schematic of stimulus forms and rotational conditions used in: (a) Experiment 1, (b) Experiment 2, and (c) Experiment 3. Actual forms were composed of pseudoshaded solid polygons.

is possible that observers employ a counting strategy and judge the angular velocity on the basis of the number of face transitions per unit time. If perceived angular velocity is thus driven by the temporal frequency of face transition, PSEs should be elevated for objects with fewer faces.

\section{Method}

Except for the differences described below, the method was the same as that in Experiment 1.

Observers. The same observers participated in all three experiments. The order of participation was randomized across observers.

Apparatus and Stimuli. The standard stimulus was identical to that used in Experiment 1. The comparison stimulus was a bisphenoid (two tetrahedrons placed base to base), which has three face transitions per rotation, one fewer than the cube. The cube and bisphenoid had approximately equal surface areas. The motion conditions, shown in Figure 2b, were similar to those used in Experiment 1: Rotations occurred about either parallel or orthogonal axes, and (for parallel axes) in either the same or the opposite direction. There was no phase manipulation since, owing to the difference in structure, there is no meaningful sense in which the forms can be said to be in phase or out of phase.

\section{Results}

The data were analyzed as in Experiment 1. The DT data for Experiment 2 are shown in Figure 5. For rotations about parallel axes, the average DT was significantly higher for same direction than for counter-rotations $\left[F(1,7)=7.38, p<.05 ; \omega^{2}=0.32\right]$. DTs were not affected by whether the axes were parallel or orthogonal. PSEs were elevated relative to POEs for all rotational con- 


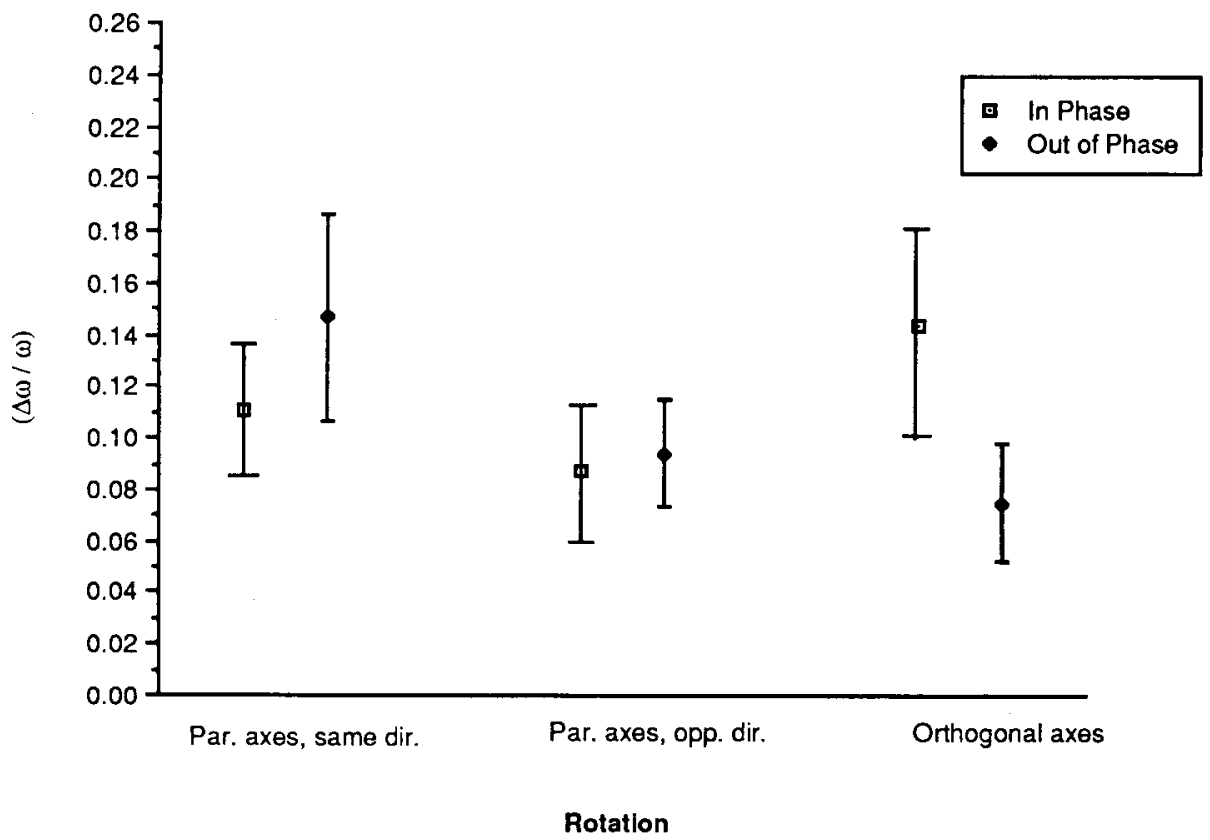

Figure 3. Mean differential thresholds for observers in Experiment 1, expressed as the difference in angular velocity $(\Delta \omega)$ divided by the standard's angular velocity $(\omega)$. Error bars indicate \pm SEM.

ditions, as is shown in Figure 6. A comparison with the PSEs obtained in Experiment 1 shows the significance of this bias $\left[F(1,7)=14.45, p<.01 ; \omega^{2}=0.51\right]$. On the average, the bisphenoid needed to rotate $9 \%$ faster than the cube to be perceived as having an equivalent angular velocity. (In order to equate temporal frequency of face transitions, the bisphenoid would need to rotate $33 \%$ faster.)

\section{EXPERIMENT 3}

Experiment 3 was designed to examine the extent to which angular velocity judgments are influenced by the velocity of the object faces. If objects of different size are employed, the two stimuli can have different velocities for corresponding elements on the objects' faces, but equivalent angular velocities. If observers base their an-

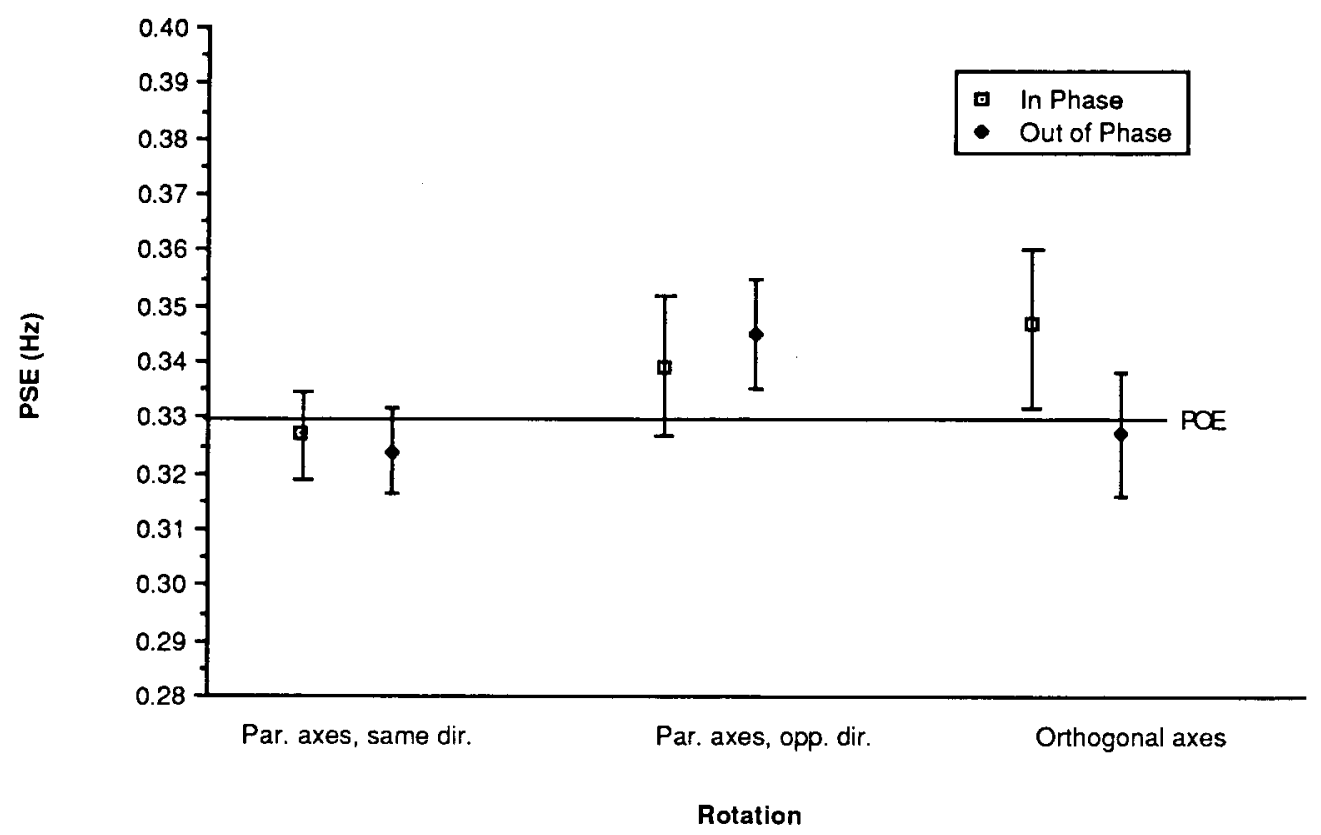

Figure 4. Mean points of subjective equality (PSEs) for observers in Experiment 1. Error bars indicate \pm SEM. Point of objective equality (POE) was .33 Hz, as indicated. 


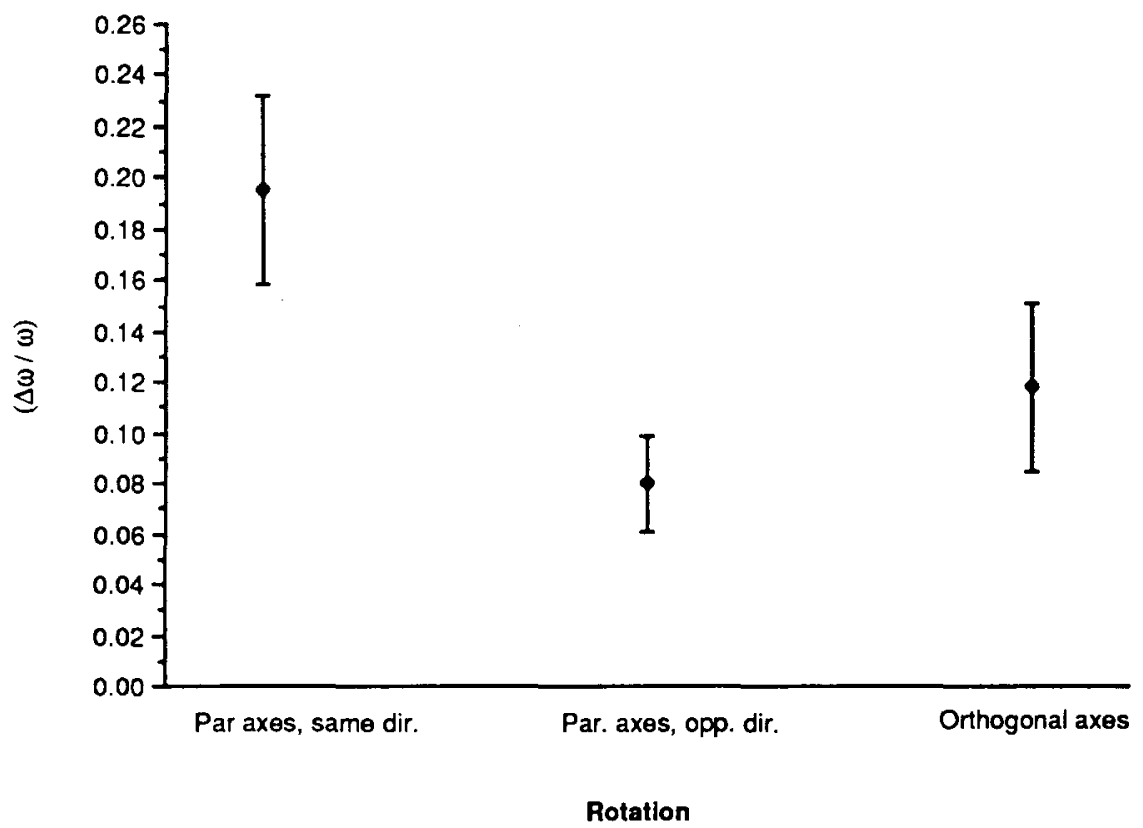

Figure 5. Mean differential thresholds for observers in Experiment 2.

gular velocity estimates on surface velocities (either in 3-D space or in the 2-D projection), angular velocities of smaller objects would be underestimated relative to a larger standard.

\section{Method}

Except as noted, the method was the same as in the previous experiments. The standard stimulus was identical to that used in Experiments 1 and 2 . The comparison stimulus was also a cube, but its edges were half the length of the standard's. The projection geometry specified that the comparison cube was a smaller object at the same depth as the standard (rather than the same size but more distant). However, it is not clear that the distortion associated with the latter interpretation was perceptible, given the viewing geometry. The rotational motion conditions, depicted in Figure 2c, were the same as those in Experiment 1.

\section{Results}

The same analyses used in Experiments 1 and 2 were applied to the data of Experiment 3. As in Experiment 1, DTs were not systematically affected by rotational con-

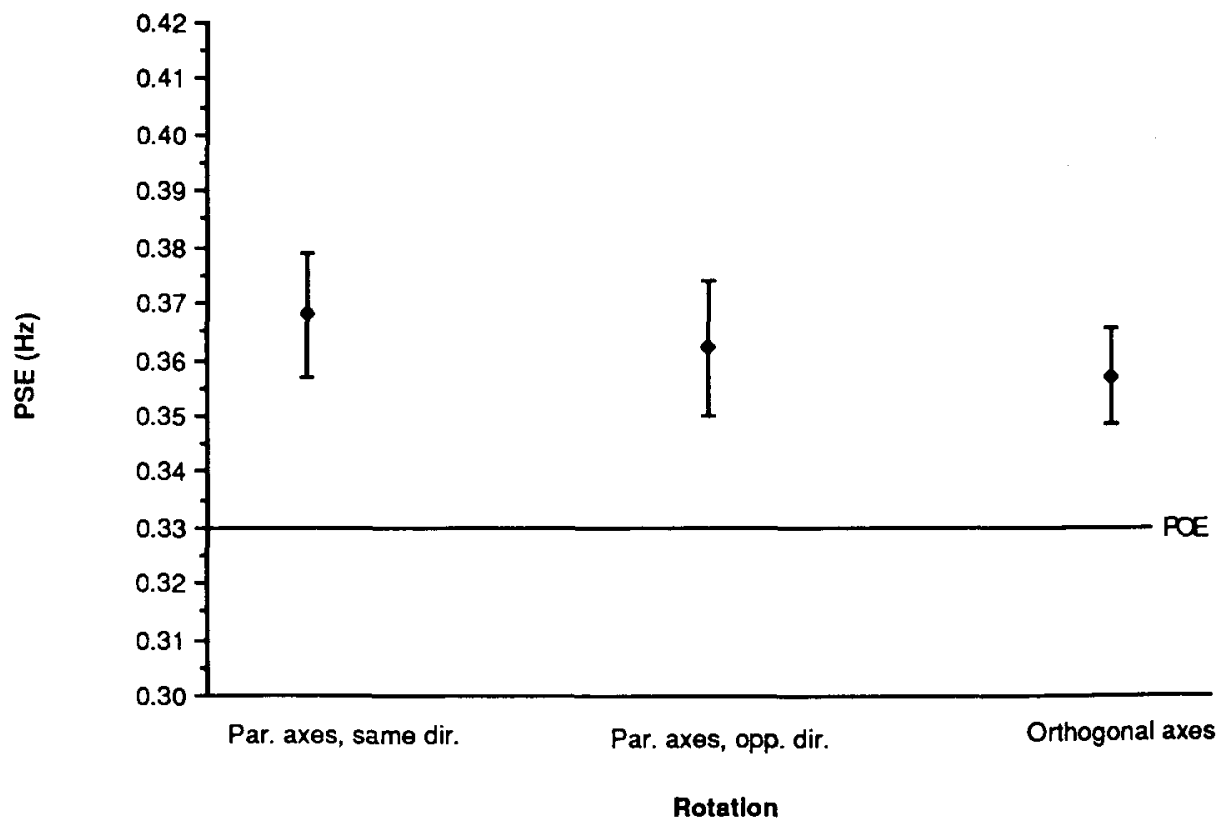

Figure 6. Mean points of subjective equality (PSEs) for observers in Experiment 2. 


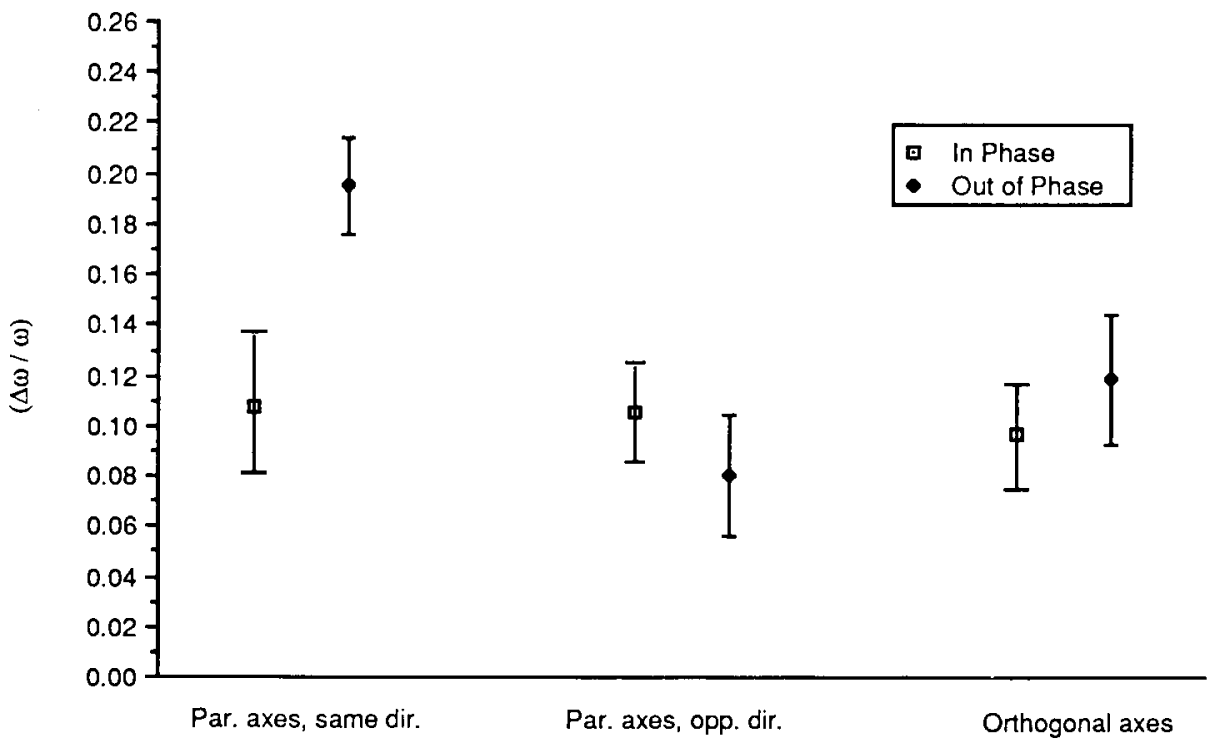

Rotation

Figure 7. Mean differential thresholds for observers in Experiment 3.

ditions or phase relation (see Figure 7). PSEs, shown in Figure 8, were consistently elevated for all conditions, as demonstrated by comparison with analogous conditions in Experiment $1\left[F(1,7)=36.29, p<.001 ; \omega^{2}=0.75\right]$. On the average, the small cube's angular velocity needed to be $18.5 \%$ greater than the large cube's to be perceived as equal. (In order to equate either 3-D space or 2-D image velocities, the smaller cube would need to rotate $100 \%$ faster.)

\section{DISCUSSION}

Across the three experiments, observers demonstrated an ability to discriminate about a $12 \%$ difference in angular velocity. This competence is near that demonstrated for linear velocities, particularly for naive observers (McKee, 1981). Furthermore, this capability is surprisingly robust for rotational conditions (i.e., whether the objects rotate about parallel or orthogonal axes, or, for

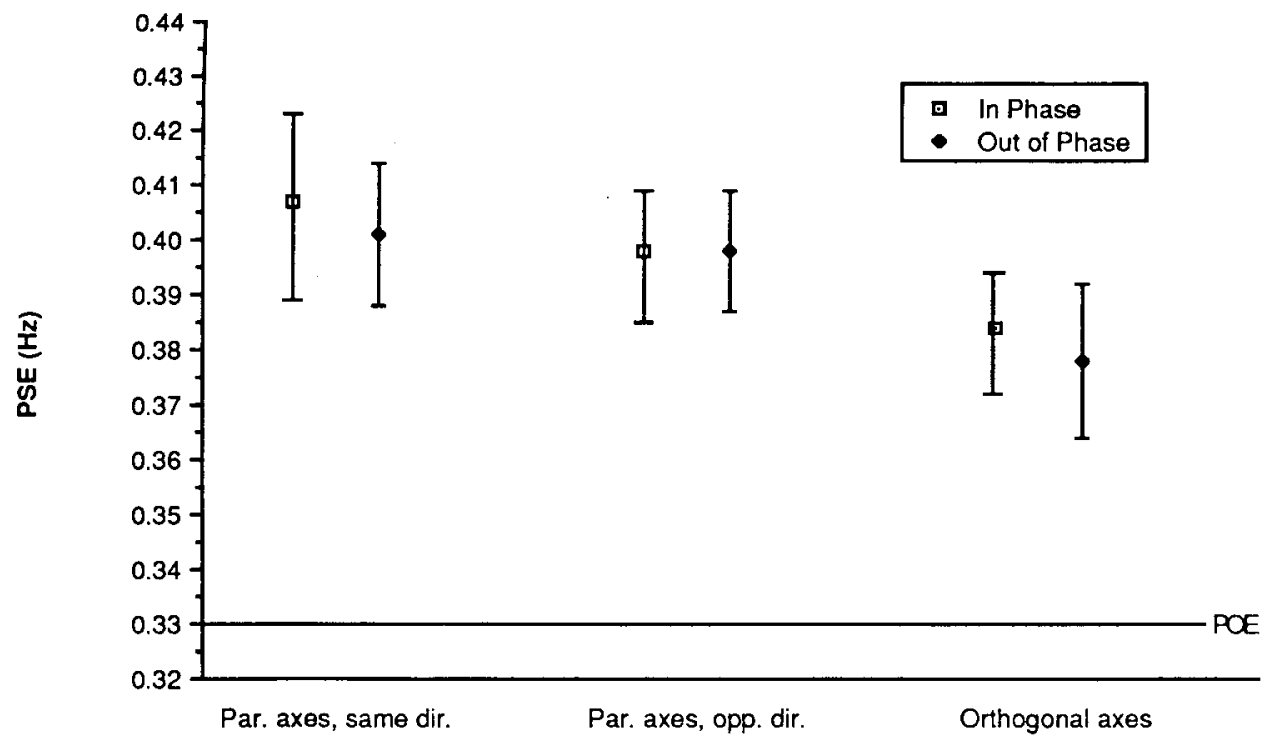

Rotation

Figure 8. Mean points of subjective equality (PSEs) for observers in Experiment 3. 
parallel axes, in the same or opposite direction) and phase relation. The only effect noted for rotational condition appeared in Experiment 2, and discrimination was actually better for counter-rotating objects.

When object properties such as shape and size were varied, the spread of discrimination functions was not affected, but perceived velocity was biased. Thus, when the comparison stimulus in Experiment 2 revealed fewer faces per rotation, PSEs were elevated. Similarly, the smaller cube in Experiment 3 needed to rotate faster than the larger standard to have an equivalent subjective velocity. In both cases, however, the observed bias only partially compensated for the difference in temporal frequency or surface velocity. To equate temporal frequency in Experiment 2 , the bisphenoid would have to rotate $33 \%$ faster than the cube. The observed $9 \%$ is thus only a $27 \%$ compensation. Similarly, the $18.5 \%$ bias noted in Experiment 3 only partially compensates for the larger cube's having surface velocities $100 \%$ greater than the smaller cube.

These findings indicate that observers' judgments correlate with three aspects of the stimulus displays: the depicted angular velocity, the depicted tangential velocity of surface elements, and other temporal factors (such as the rate of boundary deformation and edge transitions). With regard to velocity information, observers are viewing the 2-D projections of 3-D velocities. Thus, their extraction strategies may be based on the recovery of 3-D motions, or may operate directly on the 2 -D image velocities. According to most structure-from-motion algorithms, the stimuli used in these experiments provide more than sufficient information to recover the 3-D coordinates of feature points on the simulated objects, specify the axis of rotation and angular rate, and define the tangential velocities of the feature points (e.g., Ullman, 1979). ${ }^{1}$ However, aside from incidential identifications of the stimulus forms, there is no direct evidence as to whether or not observers extracted 3-D information.

A source of information for 3-D angular velocity, described in terms of the 2-D projection, is the time taken by an edge or other feature to move across the projected image. This corresponds to approximately $180^{\circ}$ of rotation (exactly $180^{\circ}$ in orthographic projection and somewhat less in perspective projection, depending on the viewing geometry). Such a strategy is similar to that proposed by Brown (1931) for linear velocity. Brown noted that perceived velocity was strongly related to the proportion of the defined motion field traversed per unit of time. For the $x$-and $y$-axis rotations used in these experiments, the boundaries of the object's projection defines a "motion field." This 2-D strategy taps a veridical source of information for angular velocity; however, it must be adapted to the sinusoidal (rather than linear) velocity function of the feature. The observer would need either to track the same feature through its entire cycle (mapping to $180^{\circ}$ of rotation), or to perform an appropriately weighted aver- aging of a sample of features. In principle, the stimuli used in the current experiments could support such strategies since, during the 4-sec exposure, each form undergoes approximately $480^{\circ}$ of rotation. The availability of information needed for these strategies could be manipulated in further studies by varying the "life" of surface features (i.e., features would only be visible for a limited number of frames rather than for the entire cycle).

The tangential velocities of surface features might also be processed in terms of their 2-D projections. However, in addition to the complication of the sinusoidal velocity function, there exists the inherent ambiguity between perceived distance and velocity. Whereas rotational velocity is not affected by perceived distance, tangential velocity (the product of rotational velocity and the distance of the feature from the axis of rotation) is. Future research can determine whether observers are influenced by 2-D image velocity or by 3-D feature velocity by using objects at different depths (cf. Rock, Hill, \& Fineman, 1968) or by exposing selected areas of the object image (to exploit the feature's constant 3-D vs. sinusoidal 2-D velocities).

The temporal factors present in the stimuli do not differ in 2-D and 3-D analysis. However, there is a confounding of two temporal cues-rate of edge transition and boundary deformation - that need to be decoupled (for example, stimuli could have color "edges" but maintain a consistent boundary shape). Stimulus patterns that support a temporal frequency pattern are found to influence perceived velocity for translational motions (Smith \& Sherlock, 1957). The temporal cues influencing angular velocity perception likewise need to be determined.

Rotational velocity, or its 2-D image correlate, is the principle determinant of angular velocity judgments in the present studies. However, the data clearly demonstrate that observers are biased by factors related to irrelevant structural characteristics of the object (e.g., surface velocities and temporal frequency cues). Still, while far from ideal, observers' competence is robust to a number of factors that would seem, a priori, to make the task more difficult (e.g., rotations about orthogonal axes, out-ofphase rotations). Furthermore, performance is comparable to that for linear velocity judgments, despite the greater complexity inherent in angular systems. This suggests that observers possess a competence for angular kinematics that is sufficient to satisfy the assumptions of most structure-from-deforming-contour algorithms. Also, people's failure to appreciate angular dynamics (Proffitt et al., in press) should not be attributed to an inability to extract angular kinematics with sufficient quantitative precision.

\section{REFERENCES}

Brown, J. F. (1931). The visual perception of velocity. Psychologische Forschung, 14, 249-268.

Cortese, J. M., Andersen, G. J. (1989). The perception of 3-D shape from deforming contours. Investigative Ophthalmology \& Visual Science, 30(Suppl.), 250. 
Lappin, J. S., Bell, H. H., Harm, O. J., \& Kottas, B. (1975). On the relation between time and space in the visual discrimination of velocity. Journal of Experimental Psychology: Human Perception \& Performance, 1, 383-394.

LEviTT, H. (1971). Transformed up-down methods in psychoacoustics. Journal of the Acoustical Society of America, 49, 467-477.

MCKEE, S. P. (1981). A local mechanism for differential velocity detection. Vision Research, 21, 491-500.

Pollick, F. E. (1989). Shape perception from dynamic occluding contours. Investigative Ophthalmology \& Visual Science, 30(Suppl.), 264.

Proffitt, D. R., Kaiser, M. K., \& Wheelan, S. M. (in press). Understanding wheel dynamics. Cognitive Psychology.

Rock, I., Hill, A. L., \& Fineman, M. (1968). Speed constancy as a function of size constancy. Perception \& Psychophysics, 4, 37-40.

Sakata, H., Shibutani, H., Ito, Y., \& Tsurugai, K. (1986). Parietal cortical neurons responding to rotary movement of visual stimulus in space. Experimental Brain Research, 61, 658-663.
Smith, O. W., \& SHERlock, C. (1957). A new explanation of the velocity transposition phenomenon. American Journal of Psychology, 70, $102-105$.

UlLMAN, S. (1979). The interpretation of visual motion. Cambridge, MA: MIT Press.

\section{NOTE}

1. Although the stimuli were produced with perspective projections, the viewing geometry is such that the resulting images closely approximate an orthographic projection. Thus, my comments and analyses will be based on this orthographic approximation, which should not cause much loss of precision or generality (Ullman, 1979).

(Manuscript received May 30, 1989; revision accepted for publication August 31, 1989.) 